\title{
Assessment of co-benefits of black carbon emission reduction measures in Southeast Asia: Part 1 emission inventory and simulation for the base year 2007
}

Didin Agustian Permadi ${ }^{1}$, Nguyen Thi Kim Oanh ${ }^{1 *}$ and Robert Vautard ${ }^{2}$

${ }^{1}$ Environmental Engineering and Management; School of Environment, Resources and Development; Asian Institute of Technology; Klong Luang, Pathumthani 12120, Thailand.

${ }^{2}$ Laboratoire des Sciences du Climate de l'Environment (LSCE), Institut Pierre Simon Laplace (IPSL), Gif Sur Yvette, France.

Correspondence to: Nguyen Thi Kim Oanh (kimoanh@ait.ac.th)

\section{Supplementary Information (SI)}

Table S1: Definition of statistical measures used for WRF/CHIMERE output evaluation.

\begin{tabular}{|c|c|c|c|}
\hline Parameters & \multicolumn{2}{|l|}{ Formula } & Suggested criteria \\
\hline Mean bias $(\mathrm{MB})^{I}$ & $M B=\frac{1}{N} \sum_{i=1}^{N}\left(M_{i}-O_{i}\right)$ & & $\begin{array}{l}\text { WS }: \leq \pm 0.5 \mathrm{~m} \mathrm{~s}^{-1} \\
\mathrm{WD}: \leq \pm 10 \mathrm{deg} \\
\mathrm{T} \quad: \leq \pm 0.5^{\circ} \mathrm{C} \\
\mathrm{H} \quad: \leq \pm 1 \mathrm{~g} \mathrm{~kg}^{-1}\end{array}$ \\
\hline $\begin{array}{l}\text { Mean absolute gross error } \\
(\mathrm{MAGE})^{I}\end{array}$ & $M A G E=\frac{1}{N} \sum_{i=1}^{N}\left|M_{i}-O_{i}\right|$ & & $\begin{array}{l}\text { WD }: \leq+30 \mathrm{deg} \\
\mathrm{T} \quad: \leq+2{ }^{\circ} \mathrm{C} \\
\mathrm{H} \quad: \leq+2 \mathrm{~g} \mathrm{~kg}^{-1}\end{array}$ \\
\hline $\begin{array}{l}\text { Root mean squared error } \\
(\mathrm{RMSE})^{l}\end{array}$ & $R M S E=\sqrt{\frac{1}{N} \sum_{i=1}^{N}\left(M_{i}-O_{i}\right)^{2}}$ & & $\mathrm{WS}: \leq+2 \mathrm{~m} \mathrm{~s}^{-1}$ \\
\hline Mean fractional bias(MFB) ${ }^{2}$ & $M F B=\frac{2}{N} \sum_{1}^{N}\left[\frac{\left(M_{i}-O_{i}\right)}{\left(M_{i}+O_{i}\right)}\right.$ & .100 & PM criteria : $\leq \pm 60 \%$ \\
\hline Mean fractional error $(\mathrm{MFE})^{2}$ & $M F E=\frac{2}{N} \sum_{1}^{N}\left[\frac{\left|M_{i}-O_{i}\right|}{\left(M_{i}+O_{i}\right)}\right.$ & .100 & PM criteria $: \leq+75 \%$ \\
\hline
\end{tabular}

Notes: Adapted from the compilation by Kim Oanh et al. (2012)

M-modeled value (model outputs for the first layer in different grids); O-observations; $\bar{O}$ : mean- observation; N-number of observations; WS-Wind Speed; WD-Wind Direction; T-Temperature; H- Relative Humidity. Sources: ${ }^{1}$ - Emery et al. (2001), ${ }^{2}$ Boylan and Russell (2006). 
Table S2: Summary of ground observations used for model evaluation.

\begin{tabular}{|c|c|c|c|c|c|}
\hline \multirow[t]{2}{*}{ No } & \multirow[t]{2}{*}{ Data sources } & \multirow[t]{2}{*}{ Data type } & \multicolumn{3}{|c|}{ Information } \\
\hline & & & Name & longitude & latitude \\
\hline \multicolumn{6}{|c|}{ Meteorological parameters } \\
\hline 1 & Olongapo-Phillippines & TMP, RH, DIR, SPD & RPLB-98426 & 14.8 & 120.3 \\
\hline 2 & Davao-Phillippines & TMP, RH, DIR, SPD & RPMD-98753 & 7.1 & 125.6 \\
\hline 3 & Don Muang-Thailand & TMP, RH, DIR, SPD & VTBD-48456 & 13.9 & 100.6 \\
\hline 4 & Trat-Thailand & TMP, RH, DIR, SPD & VTBO-48500 & 12.3 & 102.3 \\
\hline 5 & Pnom Penh-Cambodia & TMP, RH, DIR, SPD & VDPP-48991 & 11.5 & 104.8 \\
\hline 6 & Jakarta-Indonesia & TMP, RH, DIR, SPD & WIII-96749 & 6.1 & 106.7 \\
\hline 7 & Kuala Lumpur-Malaysia & TMP, RH, DIR, SPD & WMKK-48647 & 3.1 & 101.5 \\
\hline 8 & Sarawak-Malaysia & TMP, RH, DIR, SPD & WBKK-96471 & 5.9 & 116.0 \\
\hline \multicolumn{6}{|c|}{ Ground measurement of aerosols } \\
\hline 1 & AIRPET project & $\begin{array}{l}\text { Daily }(24 \mathrm{~h}) \text { average } \\
\text { of } \mathrm{PM}_{10}, \mathrm{PM}_{2.5}, \mathrm{BC} \text {, } \\
\text { and } \mathrm{OC} \text { ambient } \\
\text { concentrations }\end{array}$ & $\begin{array}{l}\text { - } \text { AIT, Thailand (sub- } \\
\text { urban) } \\
\text { - Tegallega (TL), } \\
\text { Indonesia (mix } \\
\text { urban) } \\
\text { - Manila (MO), } \\
\text { Philippines } \\
\text { observatory (mix } \\
\text { urban) } \\
\text { - Thuong Dinh (TD), } \\
\text { Vietnam (mix } \\
\text { urban) }\end{array}$ & $\begin{array}{l}100.6 \\
107.6 \\
121.1\end{array}$ & $\begin{array}{l}14.1 \\
-6.9 \\
14.6\end{array}$ \\
\hline 2 & APN project & $\begin{array}{l}\text { Hourly EC/BC and } \\
\text { OC }\end{array}$ & $\begin{array}{l}\text { AIT, Thailand (sub- } \\
\text { urban) }\end{array}$ & 100.6 & 14.1 \\
\hline 4 & $\begin{array}{l}\text { Urban air quality } \\
\text { monitoring network in } \\
\text { Bangkok, Thailand }\end{array}$ & Hourly $\mathrm{PM}_{10}$ & $\begin{array}{l}\text { EGAT (13T), Thailand } \\
\text { (mix urban) } \\
\text { Rangsit (20T), } \\
\text { Thailand (mix urban) }\end{array}$ & $\begin{array}{l}100.5 \\
100.6\end{array}$ & $\begin{array}{l}13.8 \\
13.9\end{array}$ \\
\hline 5 & $\begin{array}{l}\text { Urban air quality } \\
\text { monitoring network in } \\
\text { Kuala Lumpur, Malaysia }\end{array}$ & Hourly $\mathrm{PM}_{10}$ & $\begin{array}{l}\text { - Petaling Jaya, } \\
\text { Malaysia (Urban) } \\
\text { - Jerantut, } \\
\text { Malaysia(remote) } \\
\end{array}$ & $\begin{array}{l}101.6 \\
101.7\end{array}$ & $\begin{array}{l}3.1 \\
3.1\end{array}$ \\
\hline 6 & $\begin{array}{l}\text { City air quality } \\
\text { monitoring network in } \\
\text { Surabaya, Indonesia }\end{array}$ & Hourly $\mathrm{PM}_{10}$ & SUF1 (mix urban) & 112.7 & -7.3 \\
\hline \multicolumn{6}{|c|}{ Ground measurement of AOD } \\
\hline 1 & AERONET Thailand & AOD level 2 & Phimai & 102.6 & 15.2 \\
\hline 2 & AERONET Singapore & AOD level 2 & Singapore & 103.8 & 1.3 \\
\hline 3 & AERONET Vietnam & AOD level 2 & Bac Giang & 106.2 & 21.3 \\
\hline 4 & AERONET Vietnam & AOD level 2 & Bac Lieu & 105.7 & 9.3 \\
\hline 5 & AERONET Taiwan & AOD level 2 & Chen Kung & 120.2 & 23.0 \\
\hline 6 & AERONET Hong Kong & AOD level 2 & Hok Sui & 114.3 & 22.2 \\
\hline 7 & AERONET Thailand & AOD level 2 & Mukdahan & 104.7 & 16.6 \\
\hline 8 & AERONET Indonesia & AOD level 2 & Puspitek Serpong & 106.6 & 6.3 \\
\hline 9 & AERONET Thailand & AOD level 2 & Silpakorn University & 100.0 & 13.8 \\
\hline 10 & AERONET Thailand & AOD level 2 & Songkhla Met Station & 100.6 & 7.2 \\
\hline
\end{tabular}


Table S3: Summary of simulated $\mathrm{PM}_{10}, \mathrm{PM}_{2.5}$ and $\mathrm{BC}$ ground-level concentrations.

\begin{tabular}{|c|c|c|c|c|c|c|c|c|c|c|c|c|c|}
\hline \multirow[b]{2}{*}{ Pollutants } & \multirow[b]{2}{*}{ Averaging period } & \multicolumn{12}{|c|}{ Month, 2007} \\
\hline & & Jan & Feb & Mar & Apr & May & Jun & Jul & Aug & Sept & Oct & Nov & Dec \\
\hline \multirow{3}{*}{$\begin{array}{c}\mathrm{PM}_{10} \\
\left(\mu \mathrm{g} \mathrm{m}^{-3}\right)\end{array}$} & Max hourly & 325 & 267 & 157 & 327 & 242 & 253 & 261 & 245 & 240 & 259 & 164 & 162 \\
\hline & Max monthly avg & 69 & 89 & 65 & 127 & 62 & 48 & 72 & 58 & 69 & 86 & 44 & 30 \\
\hline & Annual average & \multicolumn{12}{|c|}{50} \\
\hline \multirow{2}{*}{$\begin{array}{c}\mathrm{PM}_{2.5} \\
\left(\mu \mathrm{g} \mathrm{m}^{-3}\right)\end{array}$} & Max hourly & 188 & 154 & 157 & 189 & 143 & 147 & 147 & 150 & 139 & 150 & 99 & 98 \\
\hline & Max monthly avg & 40 & 55 & 49 & 82 & 47 & 27 & 44 & 37 & 45 & 53 & 27 & 19 \\
\hline \multirow{3}{*}{$\begin{array}{c}\mathrm{BC} \\
\left(\mu \mathrm{g} \mathrm{m}^{-3}\right)\end{array}$} & Max hourly & 39 & 31 & 28 & 38 & 30 & 31 & 33 & 32 & 30 & 32 & 23 & 20 \\
\hline & Max monthly avg & 8.2 & 10.1 & 21.1 & 15.8 & 7.1 & 6.1 & 9.3 & 7.8 & 8.7 & 10.9 & 5.9 & 2.1 \\
\hline & Annual average & \multicolumn{12}{|c|}{6} \\
\hline
\end{tabular}




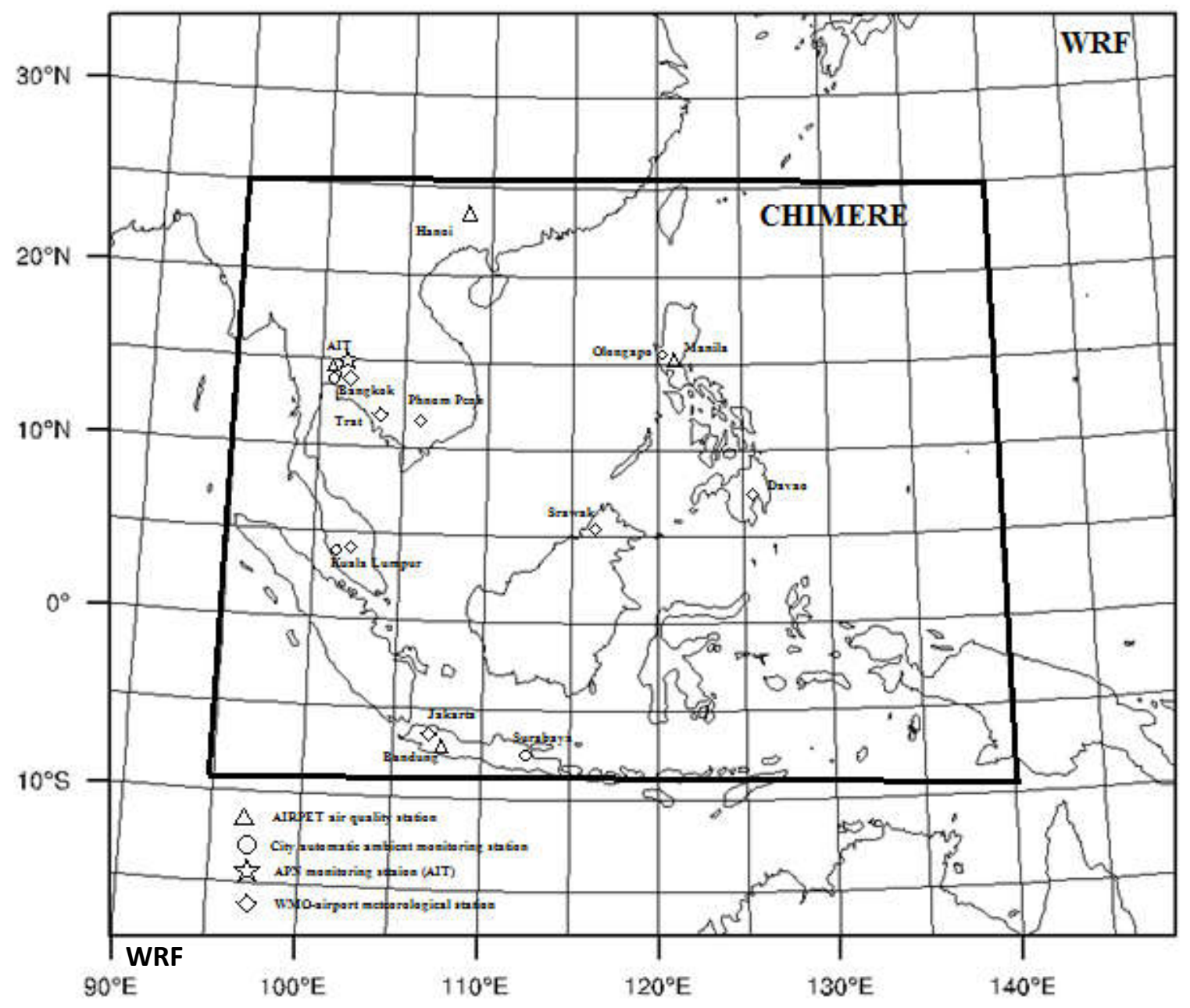

Figure S1: Modeling domains of WRF and CHIMERE. 

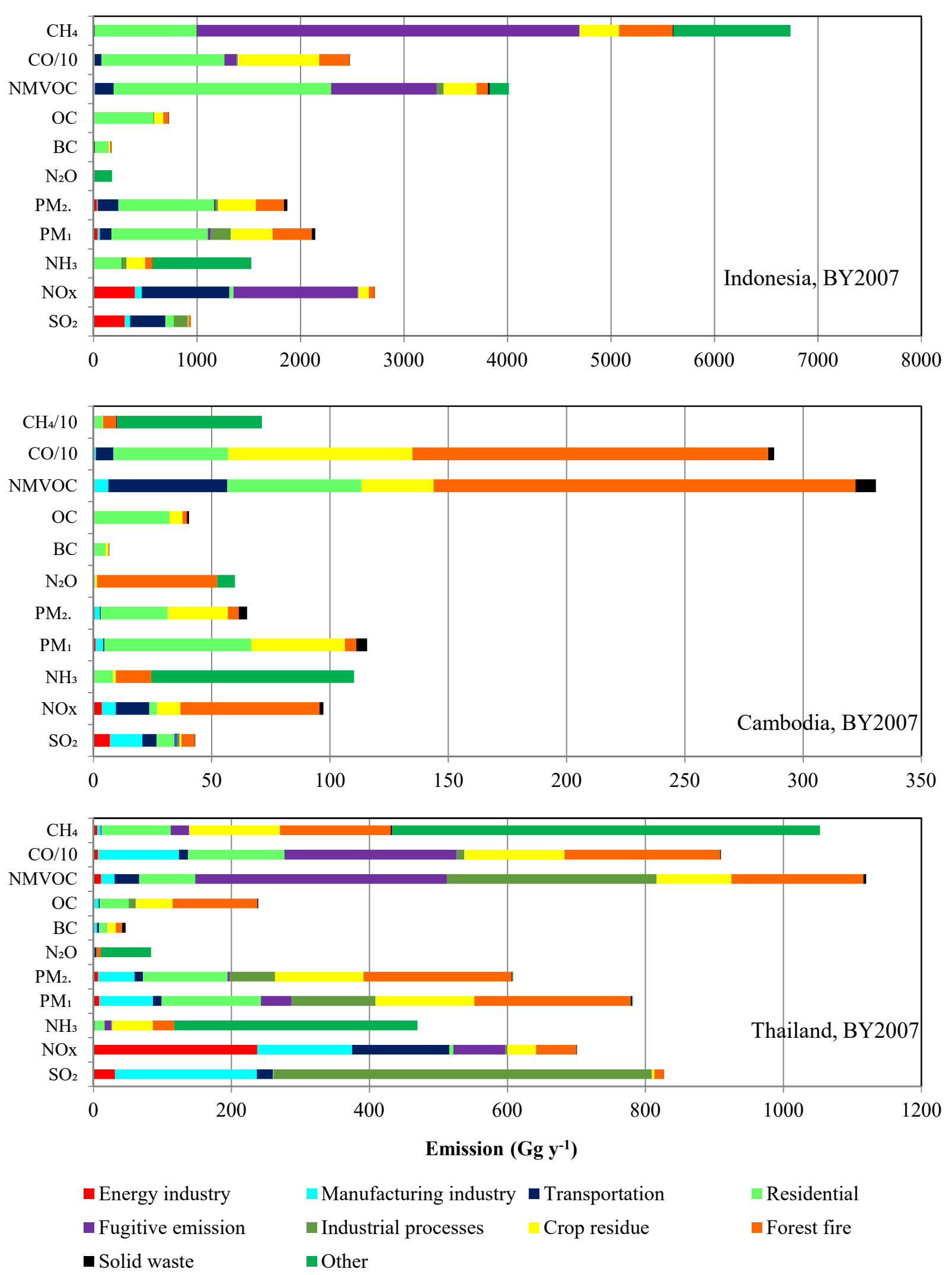

Figure S2: Total emission and shares for Indonesia, Cambodia and Thailand for base year of 2007 

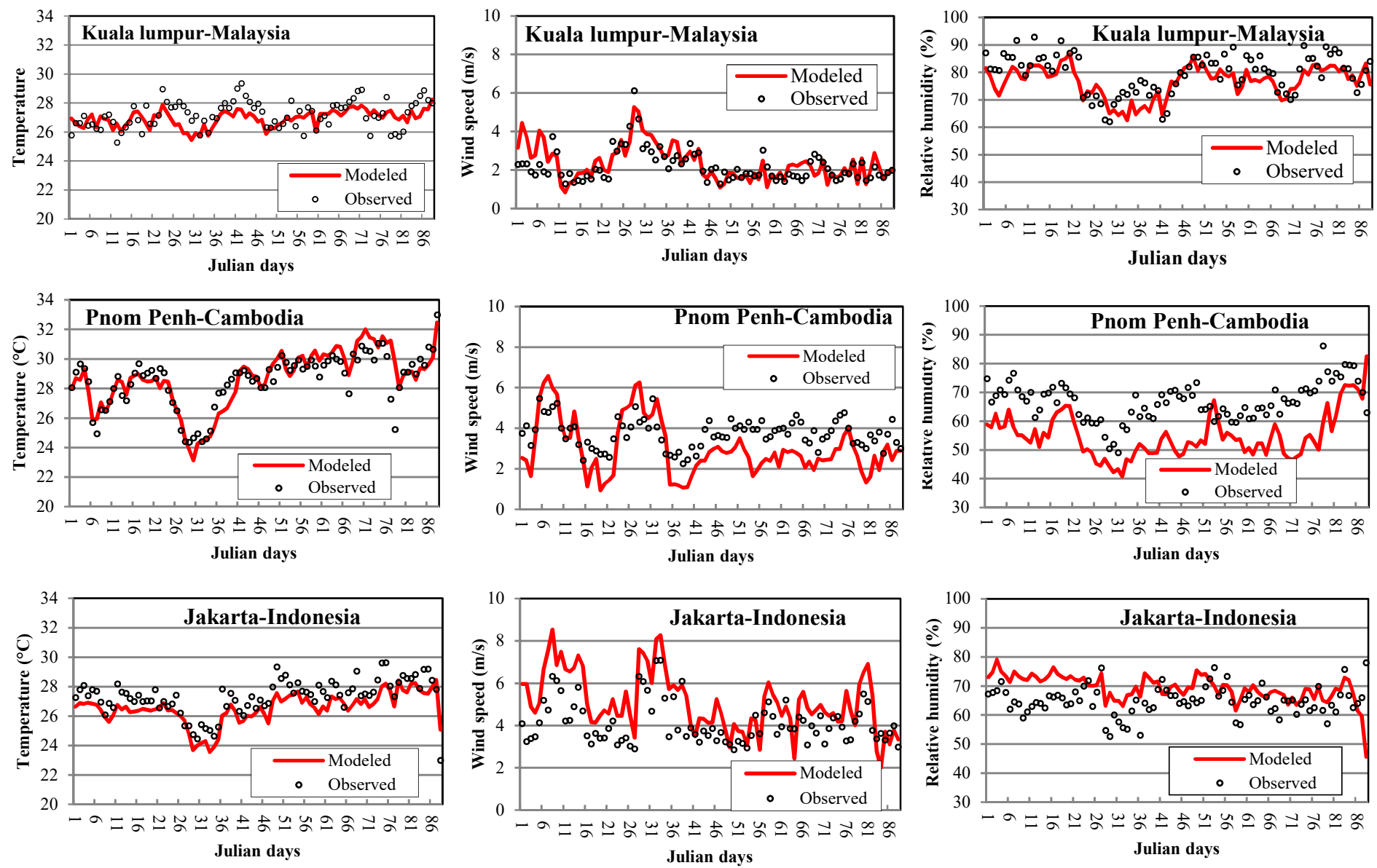

Figure S3a: Time series of daily average modeled (WRF) vs observed relative humidity, temperature and wind speed at three WMO stations in Kuala Lumpur (Malaysia), Phnom Penh (Cambodia), and Jakarta (Indonesia) for period of January - March 2007. 

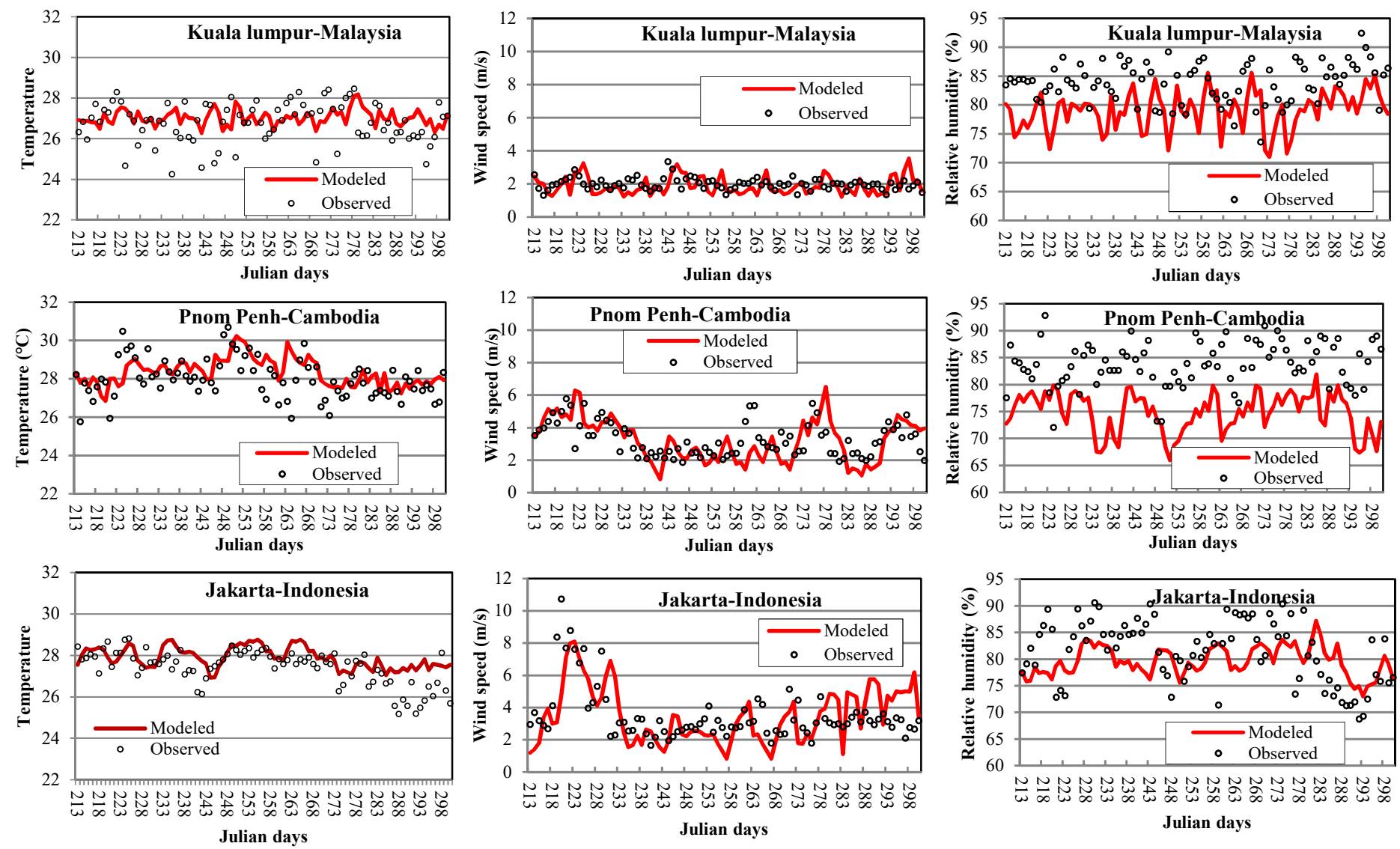

Figure S3b: Time series of daily average modeled (WRF) vs observed relative humidity, temperature and wind speed at three WMO stations in Kuala Lumpur (Malaysia), Phnom Penh (Cambodia), and Jakarta (Indonesia) for period of August - October 2007. 

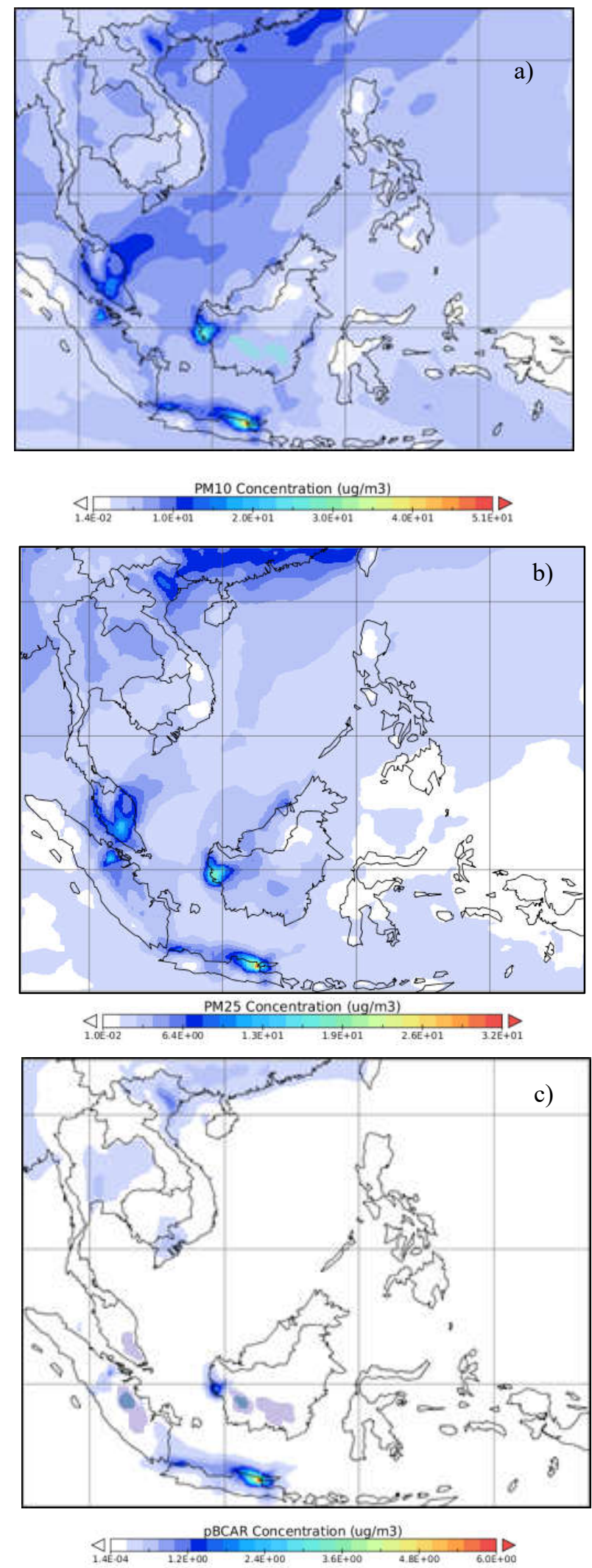

Figure S4: Simulated annual average concentration of 2007: a) $\mathrm{PM}_{10}$, b) $\mathrm{PM}_{2.5}$ and c) BC over the SEA domain. 

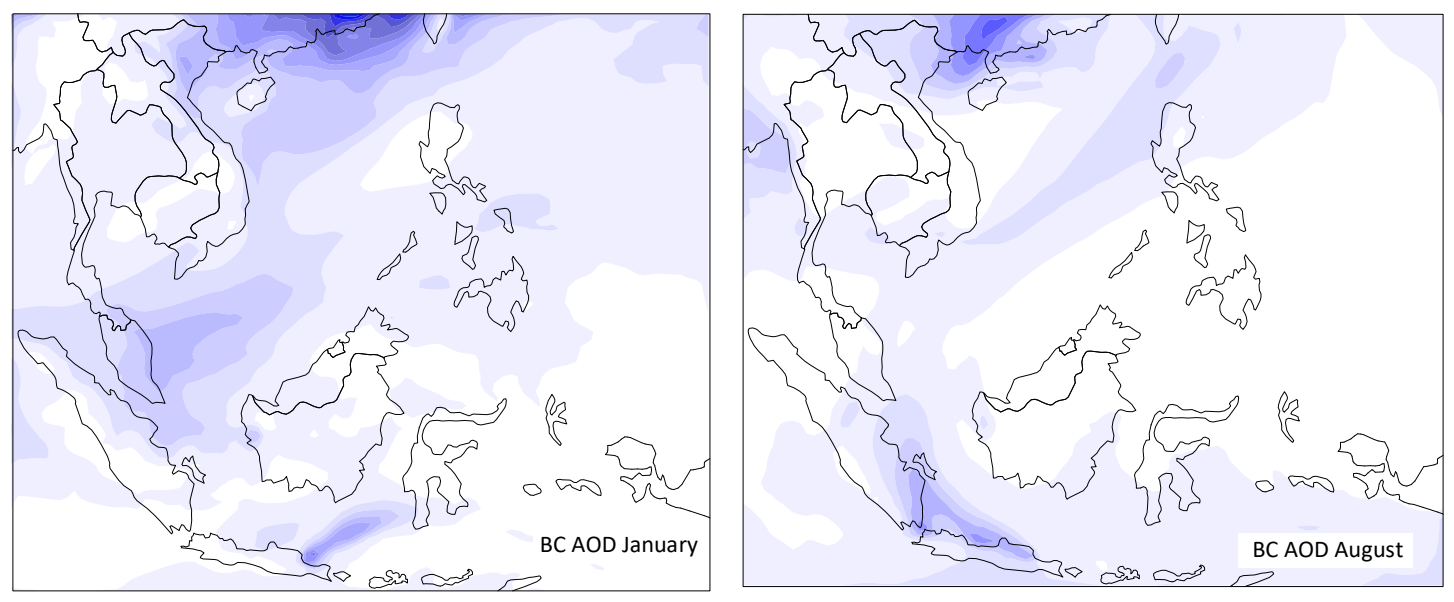

BC AOD
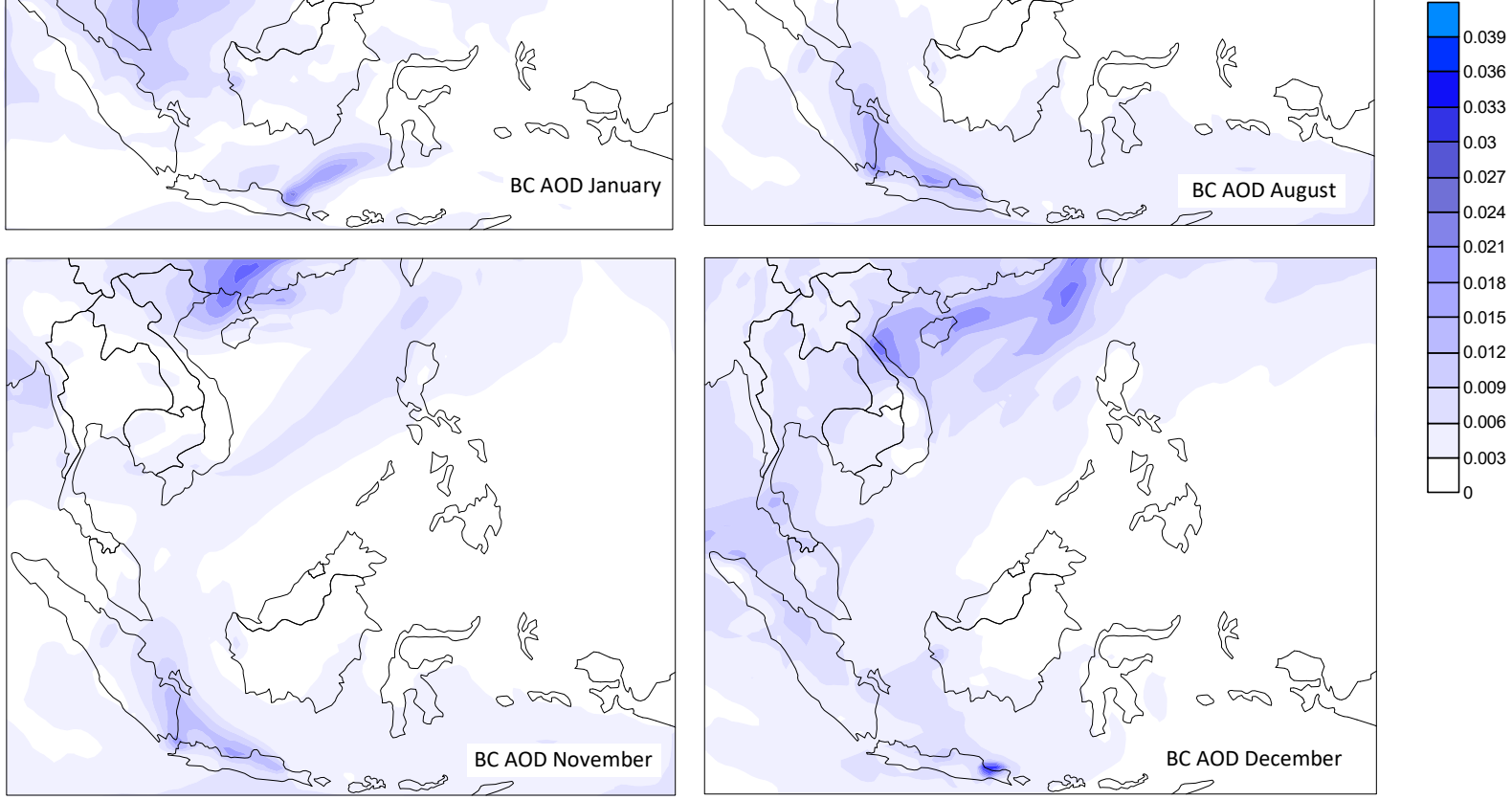

Figure S5: Monthly average of simulated BC AOD for the selected months. 